\title{
Erratum
}

J. Microbiol. Biotechnol. (2017), 27(8), 1519-1528

http://dx.doi.org/10.4014/jmb.1705.05028

\section{Erratum to: Outer Membrane Vesicles Derived from Salmonella Enteritidis Protect against the Virulent Wild-Type Strain Infection in a Mouse Model}

\author{
Qiong Liu ${ }^{1,2 \dagger}$, Jie $\mathrm{Yi}^{2 \dagger}$, Kang Liang ${ }^{2}$, Xiangmin Zhang ${ }^{3}$, and Qing Liu ${ }^{4 *}$ \\ ${ }^{1}$ Department of Medical Microbiology, School of Medicine, Nanchang University, Nanchang, China, 330006 \\ ${ }^{2}$ Institute of Preventive Veterinary Medicine, Sichuan Agricultural University, Chengdu, China, 611130 \\ ${ }^{3}$ Department of Pharmaceutical Sciences, Eugene Applebaum College of Pharmacy/Health Sciences, Wayne State University, Detroit, MI, \\ USA, 48202 \\ ${ }^{4}$ College of Animal Science and Technology, Southwest University, Chongqing, China, 400715
}

This erratum is being published to correct the error of Fig. 1B in page 1522. The previous Fig. 1B should be replaced with the below new Fig. 1B.

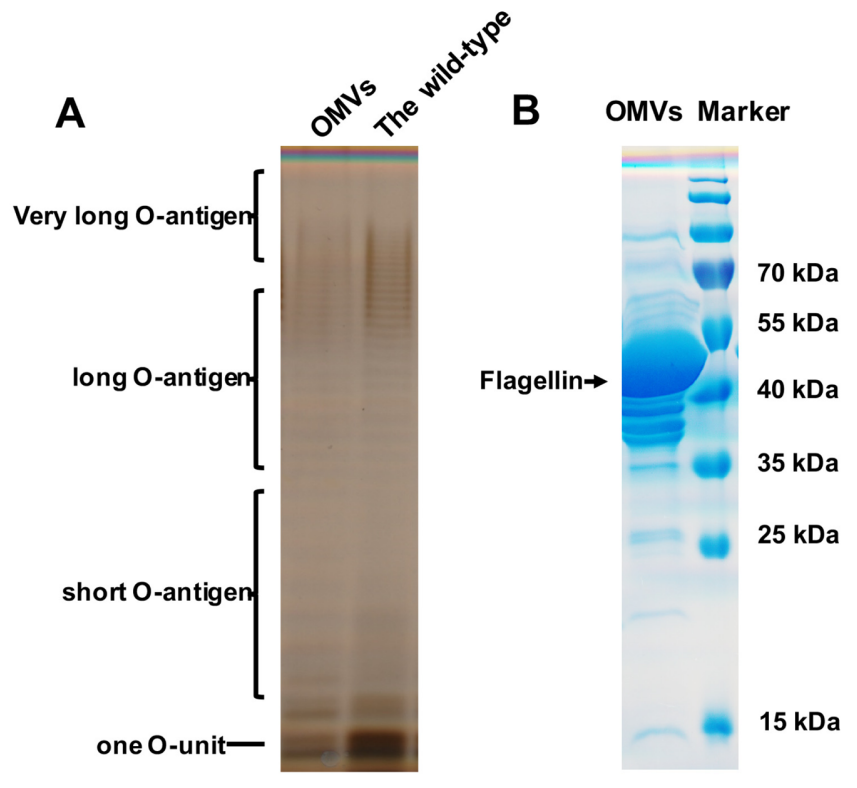

The corrected version of this article is available in the Archive (http://jmb.or.kr). 